\title{
Straddle Injuries to the Bulbar Urethra: Management and Outcome in 53 Patients
}

\author{
Mohammed Abd-alla Elgammal
}

Department of Urology, Assiut University Hospital, Assiut, Egypt

\begin{abstract}
Objective: To describe our experience with blunt injuries to the bulbar urethra and their late sequelae to identify factors that may affect patient outcome.

Materials and Methods: A retrospective study was performed on 53 male patients who presented, between January 2001 and December 2005, with blunt traumatic injury to the bulbar urethra. The definitive diagnosis of urethral rupture was made by retrograde urethrography, where urethral rupture was classified into partial or complete. The minimum follow-up period was 3 years. The initial management was either suprapubic cystostomy or endoscopic urethral realignment over a urethral catheter using a cystoscope to pass a guide-wire over which the catheter was inserted. Stricture formation was managed by visual internal urethrotomy (VIU) for passable strictures and urethroplasty (stricture excision and re-anastomosis) for impassable strictures or recurrence after VIU. The follow-up period was three years. The results were analyzed by SPSS software (chi-square and Student's-t-test).

Results: Stricture formation occurred in 19 of 22 patients (86\%) with complete urethral rupture and in 10 of $31(32 \%)$ with partial rupture $(\mathrm{p}<0.001)$. Strictures occurred in 11 of $31(35 \%)$ patients treated initially with suprapubic cystostomy and in 18 of $22(82 \%)$ treated with primary urethral realignment $(\mathrm{p}<0.001)$. The success rate after VIU was $15 \%(4$ of 26 patients) and after urethroplasty it was $96 \%$ ( 24 of 25 patients $)(p<0.001)$.

Conclusions: Suprapubic cystostomy is better than urethral realignment and catheterization as primary management after straddle injury to the bulbar urethra. Stricture excision and re-anastomosis is better than VIU as delayed management for strictures that develop after straddle injury to the bulbar urethra.
\end{abstract}

Key words: urethra; trauma; rupture: management; reconstructive surgical procedures

Int Braz J Urol. 2009; 35: 450-8

\section{INTRODUCTION}

Blunt or penetrating trauma may cause anterior urethral injuries. Blunt injuries are more commonly diagnosed, and the bulbar urethra is the most frequently injured segment (85\%) (1) because it is fixed beneath the pubic bone, unlike the freely mobile pendulous urethra. Blunt injuries to the bulbar urethra are typically caused by straddle type injuries (e.g., motor vehicle accidents; bicycle accidents; falling astride onto a fence, railing or saddle) or kicks to the perineum. The force contacting the perineum crushes the bulbar urethra against the inferior pubic rami, leading to contusion or urethral laceration (2).

Management of partial and complete disruptions after blunt trauma to the bulbar urethra remains controversial. The question which is highly debated in the literature is whether it is better to divert the urine by transurethral or suprapubic catheterization after injury to the anterior urethra $(3,4)$. Another question is whether strictures that develop after straddle injury to 
the bulbar urethra are best treated with surgical repair or endoscopic urethrotomy.

Because only a few small series have been published regarding straddle injury of the bulbar urethra, we reviewed the urological trauma database at our department to better define the clinical outcome of these injuries.

\section{MATERIALS AND METHODS}

A retrospective study was carried out on 53 male patients who presented to our trauma unit between January 2001 and December 2005 with blunt traumatic injury of the bulbar urethra. No attempts were made to catheterize the patients in the emergency room. Blood at the meatus was the single most important clinical diagnostic sign of urethral injury.

The definitive diagnosis of urethral rupture was made by retrograde urethrography. Complete urethral rupture was diagnosed on the appearance of extravasation of contrast medium in the area of the bulbar urethra outside the corpus spongiosum, with no contrast entering the prostatic urethra or bladder. Partial urethral rupture was diagnosed on the appearance of contrast extravasation in the area of the bulbar urethra within the corpus spongiosum, with contrast entering the prostatic urethra or bladder.

All patients received broad-spectrum antibiotics at induction of anesthesia. Either suprapubic cystostomy or endoscopic urethral realignment was used as primary management. In cases of endoscopic urethral realignment, a cystoscope was used to bypass the ruptured segment by introducing a guide-wire through its channel to the bladder and then a silicone catheter (F18) was passed over the guide wire. Under fluoroscopic guidance, contrast medium was injected into the catheter to verify its position inside the bladder. Suprapubic catheters were left indwelling for three weeks, then spigoted, and if patients could urinate freely, they were removed. If patients could not urinate, re-evaluation of the urethra using ascending urethrography was done. Urethral catheters after endoscopic realignment were left in place for two and three weeks in partial and complete injury, respectively. In cases of complete urethral injury with perineal hematoma or extravasation, no attempt at urethral realignment was made. All patients were instructed to return for follow-up routinely every three months in the first year then every six months thereafter. Retrograde urethrography was performed if the patient had difficulty urinating, maximum urine flow rate (Qmax) was less than $10 \mathrm{~mL} /$ minute or the postvoiding residual urine volume (PVR) was $>50$ $\mathrm{mL}$ on ultrasound. Patients with a passable stricture were managed by visual internal urethrotomy (VIU) as a trial of less invasive treatment. Patients with an impassable stricture or failed VIU were managed by open urethroplasty. Treatment success was defined as no symptoms of infravesical obstruction, Qmax persistently $>15 \mathrm{~mL} /$ second, PVR persistently $<50$ $\mathrm{mL}$ and good urethral caliber on ascending urethrography.

Statistical analysis was performed on computer software (SPSS for Windows; SPSS, Inc, Chicago, IL, USA) using the chi-square and Student's- t-test.

\section{RESULTS}

\section{Patient Characteristics}

The mean patient age was 31 (range 18 to 52 years). The mechanism of injury was a fall astride in $34(64 \%)$ of the patients, a direct kick to the perineal region in $16(30 \%)$ and a motorcar accident in $3(6 \%)$. The main complaint was bleeding per urethra in 39 patients $(74 \%)$ and inability to urinate in $14(26 \%)$. Perineal and scrotal haematoma was present in 9 patients $(17 \%)$ while perineal extravasation of urine was present in $3(6 \%)$. Partial urethral disruption was found in 31 patients $(58 \%)$ and complete urethral disruption in $22(42 \%)$. There were no patients with urethral contusion alone.

\section{Partial Urethral Disruption}

Of the 31 patients with partial urethral rupture, $19(61 \%)$ were treated primarily with suprapubic cystostomy, and stricture formation occurred in 2 $(11 \%)$ of these patients 2 and 6 months after catheter removal; 12 patients $(39 \%)$ were treated primarily with urethral realignment, and stricture formation occurred in $8(67 \%) 4-12$ months (mean $=8$ months) 
after catheter removal. Mean stricture length in patients treated primarily with suprapubic cystostomy was not statistically different from those treated primarily with urethral realignment $(1.1 \mathrm{~cm}$ range 0.9 to $1.3 \mathrm{~cm}$ and $1.3 \mathrm{~cm}$ range $1.1-1.6 \mathrm{~cm}$ respectively (p $=0.65)$. All 10 patients who developed stricture after partial disruption were treated by VIU, re-stricture developed in $6(60 \%)$ 4-9 months after VIU, and all of these patients were treated successfully with anastomotic urethroplasty (Figure-1).

\section{Complete Urethral Disruption}

Of the 22 patients with complete urethral rupture, $12(55 \%)$ were treated primarily with su- prapubic cystostomy, stricture formation occurred in 9 patients $(75 \%)$ while the suprapubic catheter was still in place in 6 patients $(67 \%)$ or within 6 months of catheter removal in 3 patients $(33 \%) ; 10$ patients $(45 \%)$ were treated primarily with urethral realignment, and stricture formation occurred in all patients $(100 \%) 4-9$ months (mean $=6$ months) after catheter removal. The duration of stricture formation was not significantly different between partial and complete urethral rupture $(\mathrm{p}=0.15)$. Mean stricture length in patients treated primarily with suprapubic cystostomy was significantly shorter than in those treated primarily with urethral realignment $(1.4 \mathrm{~cm}$ range 1.2 to 1.5 $\mathrm{cm}$ and $1.7 \mathrm{~cm}$ range 1.6 to $2.2 \mathrm{~cm}$ respectively $(\mathrm{p}=$ 0.031 ). Of the 19 patients who developed a stricture

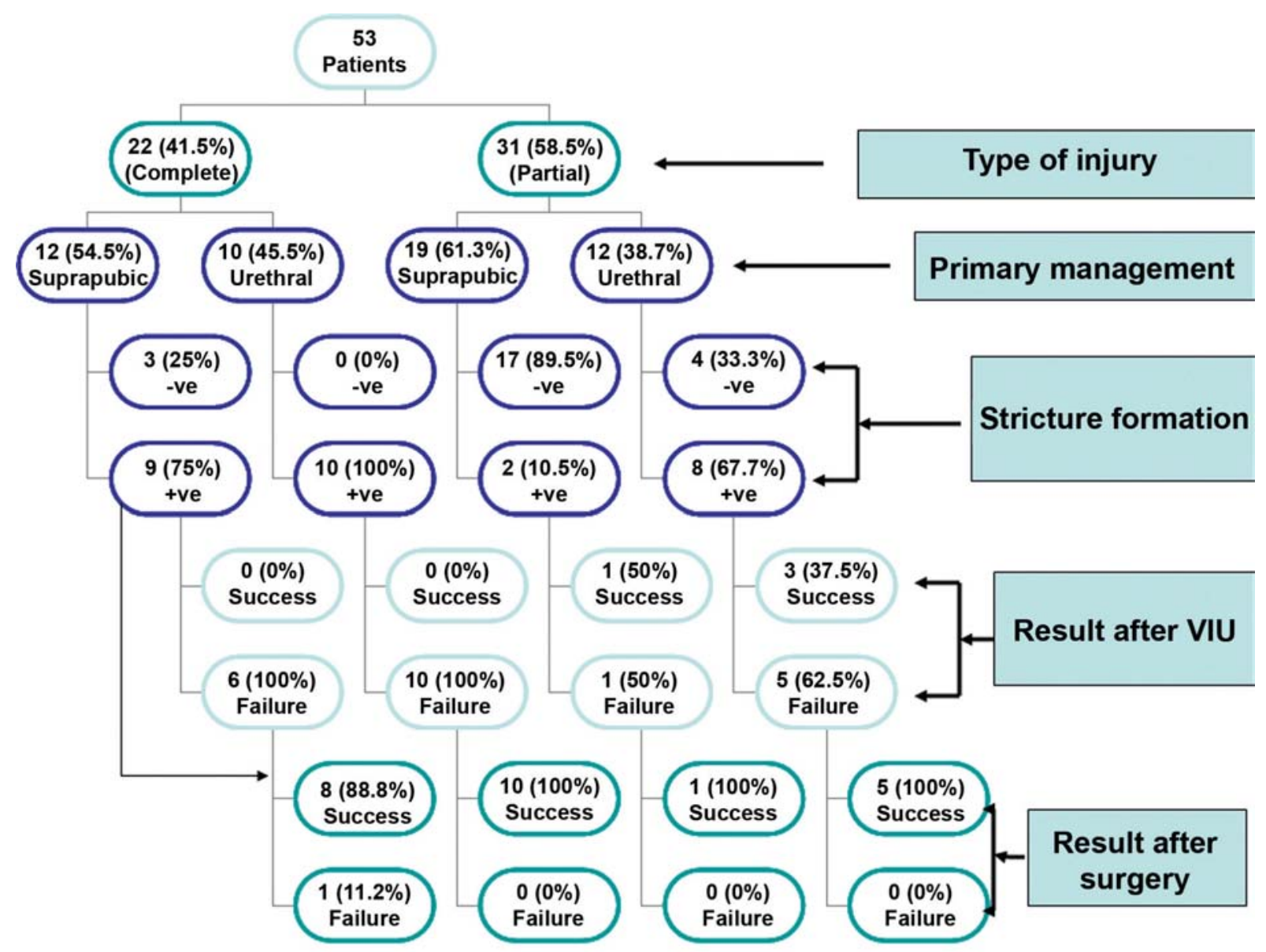

Figure 1 -Algorithm shows management outlines and outcomes in all patients. VIU = visual internal urethrotomy, -ve = no stricture formation occurred, + ve $=$ stricture formation occurred. 
Table 1 - Stricture formation in relation to type of urethral injury and primary treatment.

\begin{tabular}{llccc}
\hline & & \multicolumn{2}{c}{ Stricture Formation } \\
& & $-\mathbf{v e}$ & $+\mathbf{v e}$ & p Value \\
\hline \multirow{2}{*}{ Type of urethral injury } & Partial rupture & 21 & 10 & $<0.001$ \\
& & $68 \%$ & $32 \%$ & \\
& Complete rupture & 3 & 19 & \\
Primary management & & $86 \%$ & $<0.001$ \\
& Urethral catheterization & 4 & 18 & \\
& & $18 \%$ & $82 \%$ & \\
& Suprapubic cystostomy & 20 & 11 & \\
& & $65 \%$ & $35 \%$ & \\
\hline
\end{tabular}

$-v e=$ no stricture formation occurred; $+v e=$ stricture formation occurred)

after complete disruption, $16(84 \%)$ had a passable stricture and were treated by VIU; re-stricture developed in all these patients 2-6 months after the procedure. All 16 patients with recurrent stricture after VIU and 3 patients with impassable stricture after injury were treated with anastomotic urethroplasty, and only one of these 19 patients $(5 \%)$ required a repeat urethroplasty for recurrent stricture (Figure-1). The incidence of stricture formation after complete urethral disruption was significantly higher than after partial disruption (Table-1).

\section{Primary Management}

Patient characteristics were analyzed in relation to primary management (Table-2). There were no significant differences with regard to patients characteristics between those treated primarily with suprapubic cystostomy and those treated with primary urethral catheterization (Table-2). In the group of 31 patients $(58 \%)$ primarily treated with suprapubic cystostomy, stricture formation occurred in $11(36 \%)$, whereas in the $22(42 \%)$ treated, with primary urethral catheterization, stricture formation occurred in $18(82 \%)$. The incidence of stricture formation after primary urethral catheterization was significantly higher than after suprapubic cystostomy (Table-1).

\section{Stricture Management}

In the group of 26 patients treated with VIU, $4(13 \%)$ had a successful outcome after 3 years follow-up. In the group of 25 patients treated with anastomotic urethroplasty, $24(96 \%)$ had a successful outcome after 3 years follow-up. The difference between these treatment outcomes was statistically significant (Table-3).

Table 2 - Patient characteristics in relation to primary treatment.

\begin{tabular}{lccc}
\hline & Urethral Catheterization & Suprapubic Cystostomy & p Value \\
\hline Mean age (years) & 33 & 28 & 0.60 \\
Mean follow up (months) & 42 & 42 & 0.92 \\
Fall astride (N. of patients) & 13 & 21 & 0.51 \\
Direct trauma (N. of patients) & 7 & 9 & 0.82 \\
Motor car (N. of patients) & 1 & 2 & 0.76 \\
Hematoma (N. of patients) & 3 & 6 & 0.59 \\
\hline
\end{tabular}


Table 3 - Outcomes after urethroplasty and visual internal urethrotomy (VIU).

\begin{tabular}{cccc}
\hline & Success & Failure & p Value \\
\hline Urethroplasty & 24 & 1 & $<0.001$ \\
VIU & $96 \%$ & $4 \%$ & \\
& 4 & 26 & \\
\hline
\end{tabular}

\section{COMMENTS}

Management of partial urethral lacerations typically entails suprapubic urinary diversion or primary realignment/urethral catheterization for 1 to 2 weeks. Incomplete lacerations usually heal rapidly and with a low stricture rate. When strictures do occur, they are typically short or flimsy and can be managed effectively by VIU (5). In the present study, stricture formation developed in $11 \%$ of patients managed primarily with suprapubic cystostomy and in $67 \%$ of patients who were managed primarily with urethral realignment.

Early reports by Pontes and Pierce stated that excellent results could be obtained with urinary diversion rather than realignment $(2,4)$ but Husmann et al. reported that urethral realignment after surgical repair of the urethra achieved a better outcome (3). However, the type of trauma in the study by Husmann et al. was penetrating, whereas in the present study all patients had blunt trauma.

In the recent review of straddle injuries to the bulbar urethra by Park and McAninch, 47 of 78 patients $(60 \%)$ presented 6 months to 10 years after the acute injury with obstructive voiding symptoms or frank urinary retention (6). In the present study stricture formation developed after 4-12 months (mean = 8 months) in patients with partial urethral disruption and after 3-9 months (mean $=6$ months) in patients with complete disruption $(p=0.15)$. The difference between the present study and the series reported by Park and McAninch is that we calculated the time to stricture formation only in patients who presented acutely after urethral injury and excluded patients who presented late after trauma without previous urinary diversion or urethral realignment.
Extrapolating from the experience with posterior urethral avulsion injuries, it could be expected that early endoscopic realignment over a Foley catheter in anterior urethral injury should produce fewer strictures than suprapubic cystostomy alone. The San Francisco General Hospital group recently reported its experience with 78 straddle injuries to the bulbar urethra (6). The primary realignment group more commonly required urethroplasty than the suprapubic diversion group (100\% versus $88 \%$ ) and, moreover, complex flap or graft urethroplasty was more often required. In the current study, stricture formation developed in $75 \%$ of patients managed primarily with suprapubic cystostomy and in $100 \%$ of patients who were managed primarily with urethral realignment. Also, the strictures that developed after suprapubic cystostomy were, on average, significantly shorter than the strictures that developed after urethral realignment.

It is possible that with early suprapubic cystostomy for total or partial urethral disruption the extent of the acute and chronic inflammatory cascade would be limited and the severity of stricture formation may be mitigated. Moreover, the presence of a catheter in the area of urethral injury may cause a foreign body reaction, exacerbating the acute and chronic inflammatory cascade (6). Also, irrigation and manipulation during endoscopic realignment may cause additional injury to the ruptured urethra. However, primary realignment by minimally invasive methods has become a common contemporary management option, for posterior urethral disruption particularly at high-volume trauma centers in case of posterior urethral disruptions and the stricture rates are decreased ( $53 \%$ versus $97 \%)(7,8)$. This fact is true with regard to posterior urethral disruption, where the 
main problems are urethral distraction and misalignment but in the case of anterior urethral ruptures the urethra is maintained in alignment as it enclosed inside the corpus spongiosum and strictures that develop later on are due to subsequent spongiofibrosis.

Endoscopic procedures, such as dilation or VIU, are seldom curative. The resulting strictures are usually narrower, shorter, and can be repaired by a perineal approach. The gold standard treatment is stricture excision with primary re-anastomosis. Several medical centers report success rates exceeding 95\% (9). In the current study the success rate after VIU was $15 \%$, compared to $96 \%$ after excision and re-anastomosis. The lower success rate of VIU for urethral strictures after straddle injury may be explained by excessive peri-urethral fibrosis resulting from the blunt force acting as a hammer crushing the urethra against the anvil of the pubis.

The limitations of this study are that it is not truly randomized and longer follow-up is required.

\section{CONCLUSION}

Suprapubic cystostomy is associated with a lower stricture rate than urethral catheterization as primary management after straddle injury to the bulbar urethra. Stricture excision and re-anastomosis is associated with a lower recurrence rate than VIU as delayed management for strictures after straddle injury to the bulbar urethra.

\section{CONFLICT OF INTEREST}

None declared.

\section{REFERENCES}

1. Richter ER, Morey AF. Urethral trauma. In: Wessells HB, McAninch JW, (ed.), Urological emergencies. Totowa (NJ). Humana Press. 2005; pp. 57-69.

2. Armenakas NA, McAninch JW. Acute anterior urethral injuries: diagnosis and initial management. In: McAninch JW, Jordan GH, Carroll PR (ed.), Traumatic and reconstructive urology. Philadelphia, W.B. Saunders. 1996; pp. 543-50.
3. Husmann DA, Boone TB, Wilson WT: Management of low velocity gunshot wounds to the anterior urethra: the role of primary repair versus urinary diversion alone. J Urol. 1993; 150: 70-2.

4. Pontes JE, Pierce JM Jr: Anterior urethral injuries: four years of experience at the Detroit General Hospital. J Urol. 1978; 120: 563-4.

5. Brandes S: Initial management of anterior and posterior urethral injuries. Urol Clin North Am. 2006; 33: 87-95.

6. Park S, McAninch JW: Straddle injuries to the bulbar urethra: management and outcomes in 78 patients. $\mathrm{J}$ Urol. 2004; 171: 722-5.

7. Elliott DS, Barrett DM: Long-term followup and evaluation of primary realignment of posterior urethral disruptions. J Urol. 1997; 157: 814-6.

8. Koraitim MM, Marzouk ME, Atta MA, Orabi SS: Risk factors and mechanism of urethral injury in pelvic fractures. Br J Urol. 1996; 77: 876-80.

9. Jordan GH, Virasoro R, Eltahawy EA: Reconstruction and management of posterior urethral and straddle injuries of the urethra. Urol Clin North Am. 2006; 33: 97-109.

$\overline{\text { Accepted after revision: }}$
April 15, 2009

\section{Correspondence address:}

Dr. Mohammed Abd-alla Elgammal

Lecturer of Urology

Assiut University Hospital

Assiut, 71526, Egypt

Fax: + 0020 8833-3327

E-mail: mo_elgammal@yahoo.com 


\section{EDITORIAL COMMENT}

The main shortcoming of this retrospective, non-randomized study is that it does not provide information about the factor(s) that determined the choice of initial management by suprapubic cystostomy or urethral catheterization. Therefore, unidentified selection biases may explain the results.

Nonetheless, the findings support a number of conclusions that appear logical and explicable: (1) complete urethral rupture compared with partial rupture leads to a higher rate of stricture formation, probably because there has been more damage to the peri-urethral tissues; (2) urethral re-alignment and catheterization lead to a higher stricture rate than suprapubic cystostomy, possibly because cystoscopy causes more damage to the urethra, and infection or inflammation caused by the transurethral catheter leads to more severe fibrosis; (3) urethral stricture formation after blunt external trauma usually occurs within 9 months (mean 4 to 8 months) after the injury, similar to recurrences after VIU, where the majority also occur within 12 months; (4) VIU has a lower success rate than urethroplasty, no doubt because it does not remove fibrotic tissues, and may lead to even more spongiofibrosis.

In the Results section the authors state that, in the group of 26 patients treated with VIU, only 4 (13\%) had a successful outcome after 3 years follow-up. However, $4 / 26$ is $15 \%$, as shown in Table-3. This early stricture recurrence rate of $85 \%$ after VIU is exceptionally high, compared with the recurrence rates reported in the literature. Importantly, the recurrence rate was lower $(60 \%)$ for VIU performed for strictures after partial urethral rupture, compared with complete rupture $(100 \%)$, indicating that VIU for stricture formation after complete urethral rupture is virtually futile.

Dr. Christiaan F. Heyns
Department of Urology
University of Stellenbosch \& Tygerberg Hospital
Tygerberg, South Africa
E-mail: cfh2@sun.ac.za

management of straddle injuries to the bulbar urethra. Contrary to the findings in the posterior urethra, the authors report that whether the injuries to the bulbar urethra were partial or complete disruptions, patients managed with SPT had a lower incidence of stricture formation than those undergoing endoscopic realignment. The authors present possible reasons for these counterintuitive finding. Certainly, the pathophysiology of stricture formation in crush injuries to the bulbar urethra is quite different from that of shear injuries to the posterior membranous urethra. Perhaps this accounts for the difference in findings. Of note, the results should be evaluated with some skepticism, as this is a non-randomized study and by definition subject to selection bias. The author did not provide 
any information about the selection criteria in acute management. Urethral realignment may have been attempted in patients who were older, had more comorbidities or had more severe injuries.

Following stricture formation, the authors evaluate the success rates for urethroplasty and VIU. The success rates for primary anastomotic urethroplasty quoted at $96 \%$ are in agreement with the published literature. It is interesting to note the low success rated for VIU (15\%) for strictures that are less than $2 \mathrm{~cm}$ in length. The published success rates of VIU for bulbar urethral strictures are in the range of $42-73 \%$. The lower rates seen in the current study attests to the great degree of spongiofibrosis that occurs in the setting of crush injury. It would be interesting to know whether the initial management (SPT or realignment) affects the success rates of subsequent stricture treatments (VIU or urethroplasty). However, such a subset analysis is not possible in this series due to small numbers.

The author should be congratulated for their contribution to the published literature on urethral straddle injuries. Further studies should attempt to address similar questions in a randomized setting.

Dr. Bahaa Malaeb \& Dr. Sean P. Elliott Department of Urology Surgery University of Minnesota Minneapolis, Minnesota, USA E-mail: selliott@umn.edu

\section{EDITORIAL COMMENT}

The primary responsibilities of the urologist when encountering a patient with an acute anterior urethral injury are 1) to diagnose the extent of the injury and 2) to divert the urine away from the injury if appropriate, whether it is with urethral catheterization or suprapubic diversion (1). Many times, the initial evaluation includes cystourethroscopy, which serves to both visualize the injury and to then aid in urethral catheterization, usually over a cystoscopically placed wire. Conventional wisdom would say that having a catheter across a urethral injury would augment appropriate urethral healing and subsequently decrease urethral stricture rates. This is why, for example, a catheter is nearly universally placed after radical retropubic prostatectomy or urethral reconstructive surgery. However, the superiority of suprapubic tube placement over urethral catheterization, reported by Abd-alla Elgammal in this manuscript, challenges that notion.

As discussed in the manuscript, this finding has been reported before. Park et al. analyzed a series of anterior urethral injuries from San Francisco General Hospital, and found that while strictures requiring intervention were high regardless of initial management ( $88 \%$ for suprapubic diversion v 100\% for urethral catheterization, $p=0.37$ ), the group that initially had suprapubic diversion without urethral manipulation were less likely to require complex reconstruction (2). The authors in the current study report similar findings. Patients with complete anterior urethral disruption managed with a urethral catheter had a $100 \%$ stricture rate versus a $75 \%$ stricture rate with suprapubic management. In partial disruptions, the results were even more dramatic, with strictures developing in $68 \%$ of men managed with urethral catheterization versus only $11 \%$ in men with suprapubic tubes.

What is the mechanism for the differences found in this study? If the reported outcomes are to be believed, a multi-factorial explanation is most plausible. As suggested in the manuscript, it is possible that the initial cystoscopic exam with subsequent urethral catheterization could propagate the original injury. The catheter, being a foreign body, could also worsen the periurethral inflammatory reaction and hence, scar formation (3). Finally, when a urethral 
catheter is in place, it serves to stent open the bladder neck, which can allow for leakage of urine around the catheter, further urinary extravasation through the urethral injury, and again, a worse periurethral inflammatory reaction.

So should all anterior urethral injuries be managed with suprapubic tube alone? Unfortunately, without a controlled, randomized study this question cannot be completely answered. However, the findings from this study should make all urologists question the need for heroic attempts at endoscopic realignment of an anterior urethral injury, as outcomes in terms of future stricture rates are at least similar and at worst, much higher, when placing a urethral catheter than when simply placing a suprapubic tube.

\section{REFERENCES}

1. Chapple C, Barbagli G, Jordan G, Mundy AR, Rodrigues-Netto N, Pansadoro V, et al.: Consensus statement on urethral trauma. BJU Int. 2004; 93: 1195-202.

2. Park S, McAninch JW: Straddle injuries to the bulbar urethra: management and outcomes in 78 patients. $\mathrm{J}$ Urol. 2004; 171: 722-5.

3. Talja M, Korpela A, Järvi K: Comparison of urethral reaction to full silicone, hydrogen-coated and siliconised latex catheters. Br J Urol. 1990; 66: 652-7.

Dr. Bradley A. Erickson \& Dr. Christopher M. Gonzalez Department of Urology Feinberg School of Medicine Northwestern University Chicago, Illinois, USA E-mail:j-meeks@md.northwestern.edu 